\title{
A bivariate optimal replacement policy with cumulative repair cost limit under cumulative damage model
}

\author{
MIN-TSAI LAI ${ }^{1, *}$ and SHIH-CHIH CHEN ${ }^{2}$ \\ ${ }^{1}$ Department of Business Administration, Southern Taiwan University of Science and Technology, Tainan, \\ Taiwan, R.O.C. \\ ${ }^{2}$ Department of Accounting Information, Southern Taiwan University of Science and Technology, Tainan, \\ Taiwan, R.O.C. \\ e-mail: lai1962@stust.edu.tw
}

MS received 21 November 2014; revised 15 January 2016; accepted 29 January 2016

\begin{abstract}
In this paper, a bivariate replacement policy $(n, T)$ for a cumulative shock damage process is presented that included the concept of cumulative repair cost limit. The arrival shocks can be divided into two kinds of shocks. Each type-I shock causes a random amount of damage and these damages are additive. When the total damage exceeds a failure level, the system goes into serious failure. Type-II shock causes the system into minor failure and such a failure can be corrected by minimal repair. When a minor failure occurs, the repair cost will be evaluated and minimal repair is executed if the accumulated repair cost is less than a predetermined limit $L$. The system is replaced at scheduled time $T$, at $n$-th minor failure, or at serious failure. The long-term expected cost per unit time is derived using the expected costs as the optimality criterion. The minimum-cost policy is derived, and existence and uniqueness of the optimal $n^{*}$ and $T^{*}$ are proved. This bivariate optimal replacement policy $(n, T)$ is showed to be better than the optimal $T^{*}$ and the optimal $n^{*}$ policy.
\end{abstract}

Keywords. Shock model; cumulative damage model; cumulative repair cost limit; preventive maintenance model.

\section{Introduction}

Most production systems are repaired or replaced when they have already failed. However, they may require much time and high expenses to repair a failed system, so maintaining a system to prevent failures is essential for the factory operation. The effect of an optimal preventive maintenance $(\mathrm{PM})$ policy on system's overall reliability, availability, downtime and cost of operation is well known. PM includes activities such as partial or complete overhauls at specified periods, oil changes, and lubrication.

In some practical situations, most systems would deteriorate with time and are influenced by external shocks. The level of deterioration of the system is represented by a degradation process such as corrosion, wear, material fatigue, and fatigue-crack growth in engineering applications or markers of health status and quality of life data in medical settings. Cumulative damage models are often used to describe the above situations. These models, which play an important role in reliability theory, are considered to be a sequence of shocks that occur randomly in time and cause some amount of damage to the system. These damages are additive and the system suffers failure finally by causes of

*For correspondence additive damage. Nakagawa [1] summarised PM policies and the optimization problems of these policies for cumulative damage models. The replacement models in which a system is replaced when the total damage exceeds a threshold level $k$ were proposed by Feldman [2], Nagakawa [3] and Satow et al [4]. The replacement models in which a unit is replaced at a planned time $T$ were proposed in Taylor [5], Mizuno [6], Nakagawa [1, 7], Qian et al [8] and Perry [9]. Furthermore, the replacement models in which a system is replaced after a shock $N$ were proposed in Nagakawa [10]. The replacement model with multiple decision variables $T, N$, and $k$ were proposed in Nakagawa and Kijima [11] and Satow and Nakagawa [12].

Kijima and Nakagawa [13] considered a cumulative damage shock model with an imperfect PM policy. Qian et al [8] presented an extended cumulative damage model with two types of shocks: one type is failure shock, and the other type is damage shock, from which the system suffers only damage. A periodic replacement policy was proposed and applied to the backup of files in a database system. Satow et al [4] considered a replacement problem of a cumulative damage model that deteriorates with time at a constant rate $a$. The replacement model in which a system is replaced when the total damage exceeds a threshold $k$ was proposed. Qian et al [14] considers an extended 
cumulative damage model with maintenance at each shock and minimal repair at each failure. The optimal values $T^{*}$ and $N^{*}$ that minimize the expected cost are obtained. Qian et al [15] applied a cumulative damage model for a used system with an initial damage level. Ito and Nakagawa [16] compared the standard cumulative damage model with two other cumulative damage models: (1) the amount of damage due to shocks is measured only at periodic time; and (2) the amount of damage increases linearly with time. Zhao et al [17] considered a periodic replacement model for a system that is subject to additive and independent damages simultaneously.

Lai [18] introduced the concept of a cumulative repair cost limit, which included the information of all repair costs, into the replacement model to decide whether the system should be repaired or replaced. This paper overcame the shortcomings of the traditional repair-cost-limit policy. Following the work of Lai [18], Chien et al [19] extended the work of Lai [18] by introducing the random lead time for replacement delivery. Chien et al [20] modified the work of Chien et al [19] by adding an agedependent type of failure. Chang et al [21] presented a model to determine the optimal number of minimal repairs before replacement. Chang et al [22] modified the work of Chang et al [21] by allowing an age-dependent failure type. Sheu et al [23] presented a generalised model to determine the optimal replacement policy based on multiple factors (or more information) such as the number of minimal repairs before replacement and the cumulative repair cost limit. Sheu et al [24] presented an age replacement policy for a system subject to non-homogeneous pure birth process (NHPBP) shocks. Sheu et al [25] extended the work of Sheu et al [24] and considered a bivariate replacement policy $(n, T)$ for a system subject to NHPBP shocks.

This paper considers a bivariate replacement policy ( $n$, $T$ ) with a cumulative repair cost limit in which the system is subject to a cumulative damage process. The rest of the paper is organized as follows: section 2 presents the model formulation, the long-term expected costs per unit time and the optimization. In section 3, special cases of our model are introduced. A computational example is provided to demonstrate the above results in section 4 . Section 5 concludes the paper.

\section{System description and model formulation}

We consider a system that is subject to shocks of a nonhomogeneous Poisson process (NHPP). The arrival shocks can be divided into two kinds of shocks. Each type-I shock causes a random amount of damage and these damages are additive. When the total damage exceeds a failure level, the system goes into serious failure. Type-II shock causes the system into minor failure and such a failure can be corrected by minimal repair. When a minor failure occurs, the repair cost will be evaluated and minimal repair is executed if the accumulated repair cost is less than a predetermined limit $L$. We focus on the following multi-criteria replacement policy, the system is replaced at scheduled time $T$, at the $n$-th minor failure, or at serious failure. This problem is to find the optimal $n^{*}$ and $T^{*}$ that minimizes the long-term expected cost per unit time $C(n, T, L)$ in the steady-state case.

\subsection{General model}

Suppose that shocks occur randomly according to an NHPP $\{N(t)\}_{t \geq 0}$ with intensity function $\lambda(t)$, and let $T_{1}, T_{2}, \ldots$ denote the successive arrival times of shock process. The two types of shock are associated at random with the event epochs of the shock process. At time $T_{i}$, the system has two possible types of shock. The outcome indicator function of the shock process is

$$
\eta_{i}=\left\{\begin{array}{ll}
1 & \text { type }- \text { I shock with probability } p\left(T_{i}\right) \\
0 & \text { type }- \text { II shock with probability } q\left(T_{i}\right)
\end{array} .\right.
$$

Let $N_{1}(t)=\sum_{i=1}^{N(t)} \eta_{i}$ count the number of type-I shocks of the system in the interval $[0, t]$. Then $N_{2-}$ $(t)=N(t)-N_{1}(t)$ counts the number of type-II shocks, and it can be shown that $\left\{N_{1}(t)\right\}_{t \geq 0}$ and $\left\{N_{2}(t)\right\}_{t \geq 0}$ are independent non-homogeneous Poisson processes with intensity functions $p(t) \lambda(t)$ and $q(t) \lambda(t)$ (see, e.g. Savits [26]). This is similar to the classical decomposition of a Poisson process for a constant event probability $p$.

The type-I shocks whenever occur cause some damage to the system and these damages are additive. The random amount of damage $D_{i}$ due to $i$-th type-I shock has a probability distribution $H_{i}(d)=P\left(D_{i} \leq d\right)$ and a finite mean $\mu_{d}$, $i=1,2,3, \ldots$. Then the accumulated damage till the $j$-th type-I shock after the installation $W_{j}=\sum_{i=1}^{j} D_{i}$ has the distribution function:

$$
\begin{aligned}
P\left(W_{j} \leq w\right) & =H^{(j)}(w) \\
& = \begin{cases}1 & j=0 \\
H_{1} * H_{2} * \cdots * H_{j}(w), & j=1,2,3, \cdots\end{cases}
\end{aligned}
$$

where the "*” mark is denoted the Stieltjes convolution. The probabiliity that the number of type-I shocks occurred in $[0, t]$ equals $j$ is given by

$$
P\left(N_{1}(t)=j\right)=\frac{\left(m_{1}(t)\right)^{j} \exp \left(-m_{1}(t)\right)}{j !}=P_{1, j}(t),
$$

where $m_{1}(t)=\int_{0}^{t} p(x) \lambda(x) \mathrm{d} x$ denote the mean number of type-I shocks occurred in $[0, t]$.

A serious failure will occur when the accumulated damage exceeds a failure level $K$ and the probability that serious failure will occur at the occurrence of $i$-th type I shock is $\overline{P_{i}}=H^{(i-1)}(K)-H^{(i)}(K)$. Let random variable $Z$ 
denote the occurrence time of the first serious failure, so the survival function of $Z$ is given by

$$
\begin{aligned}
\overline{F_{z}}(t) & =P(Z>t)=P\left(W_{N_{1}(t)}<K\right) \\
& =\sum_{i=0}^{\infty} P\left(N_{1}(t)=i, W_{i}<K\right)=\sum_{i=0}^{\infty} P_{1, i}(t) H^{(i)}(K)
\end{aligned}
$$

and the density function of $Z$ is

$f_{z}(t)=p(t) \lambda(t)\left(\sum_{i=0}^{\infty} H^{(i)}(K) P_{1, i}(t)-\sum_{i=1}^{\infty} H^{(i)}(K) P_{1, i-1}(t)\right)$.

Secondly, a type-II shock whenever occurs makes the system into minor failure and such a failure can be corrected by minimal repair. Hence, the probability that the number of minor failures occurred in $[0, t]$ equals $j$ is given by

$$
P\left(N_{2}(t)=j\right)=\frac{\left(m_{2}(t)\right)^{j} \exp \left(-m_{2}(t)\right)}{j !}=P_{2, j}(t)
$$

where $m_{2}(t)=\int_{0}^{t} q(x) \lambda(x) \mathrm{d} x$ denote the mean number of minor failures occurred in $[0, t]$.

Moreover, let $S_{2, j}, \quad j=0,1,2,3, \ldots$ denote the occurrence time of $j$-th minor failure, where $S_{2,0}=0$, then the distribution function of $S_{2, j}$ is given by

$$
P\left(S_{2, j} \leq t\right)=P\left(N_{2}(t) \geq j\right)=\sum_{i=j}^{\infty} P_{2, i}(t), \quad j=1,2,3, \ldots,
$$

and the density function of $S_{2, j}$ is

$f_{s_{2, j}}(t)=\frac{d}{\mathrm{~d} t} P\left(S_{2, j} \leq t\right)=\frac{d}{\mathrm{~d} t} P\left(N_{2}(t) \geq j\right)=q(t) \lambda(t) P_{2, j-1}(t)$.

When a minor failure occurs, the repair cost is evaluated. The repair cost $X_{i}$ due to $i$-th minor failure is a non-negative i.i.d. random variable with a probability distribution function $G(x)=P\left(X_{i} \leq x\right)$ and a finite mean $\mu_{x}, i=1,2,3, \ldots$ Then, the accumlated repair cost till the $j$-th minor failure $Y_{j}=\sum_{i=1}^{j} X_{i}$ has a distribution function

$$
\begin{aligned}
P\left(Y_{j} \leq y\right) & =G^{(j)}(y) \\
& =\left\{\begin{array}{ll}
1 & j=0 \\
G_{1} * G_{2} * \cdots * G_{j}(y), & j=1,2,3, \ldots
\end{array} .\right.
\end{aligned}
$$

If the accumulated repair cost exceeds a predetermined limit $L$, then the system is preventively replaced at this minor failure. Let random variable $U$ denote the occurrence time when the accumulated repair cost exceeds a predetermined limit $L$, so the survival function of $U$ is given by

$$
\begin{aligned}
\overline{F_{u}}(t) & =P(U>t)=P\left(Y_{N_{2}(t)}<L\right) \\
& =\sum_{j=0}^{\infty} P\left(N_{2}(t)=j, Y_{j}<L\right)=\sum_{j=0}^{\infty} P_{2, j}(t) G^{(j)}(L)
\end{aligned}
$$

and the density function of $U$ is $f_{u}(t)=q(t) \lambda(t)$ $\sum_{j=0}^{\infty}\left(G^{(j)}(L)-G^{(j+1)}(L)\right) P_{2, j}(t)$.

\subsection{Different replacement cases}

In this replacement model, replacements and repairs are executed according to the following scheme. Preventive replacement is carried out at scheduled time $T$, at $n$-th minor failure, or at the occurrence time of one minor failure, in which the accumulated repair cost exceeds a predetermined limit $L$. Failure replacement is executed at serious failure. If the accumulated repair cost till the $i$-th $(i<n)$ minor failure is less than $L$, then this minor failure is corrected by minimal repair. Therefore, the replacement of the system can occur at four different cases and the probabilities of four cases are derived as follows.

First, if the number of minor failures occurred in $[0, T]$ equals $j(<n)$ and the accumulated repair cost till the $j$-th minor failure is less than $L$, and there is no serious failure occurred in $[0, T]$, then preventive replacement is carried out at scheduled time $T, j=0,1,2, \ldots, n-1$. Therefore, the probability of the first case is given by

$$
\begin{aligned}
P\left(Z>T, U>T, S_{2 n}>T\right)= & \sum_{i=0}^{\infty} P_{1, i}(T) H^{(i)}(K) \\
& \times \sum_{j=0}^{n-1} P_{2, j}(T) G^{(j)}(L) \\
= & \overline{F_{z}}(T) \sum_{j=0}^{n-1} P_{2, j}(T) G^{(j)}(L) .
\end{aligned}
$$

Second, if the $n$-th minor failure occurred before time $T$ and the accumulated repair cost till the $(n-1)$-th minor failure is less than $L$, and the first serious failure occurs after time $S_{2 n}$, then the system will be replaced at the $n$-th minor failure. So, the probability of the second case is given by

$$
\begin{aligned}
P( & \left.S_{2 n}<T, Y_{n-1}<L, Z>S_{2 n}\right) \\
& =\int_{0}^{T} P\left(Y_{n-1}<L\right) \bar{F}_{z}(t) f_{S_{2 n}}(t) \mathrm{d} t \\
& =G^{(n-1)}(L) \int_{0}^{T} \bar{F}_{z}(t) P_{2 n-1}(t) q(t) \lambda(t) \mathrm{d} t .
\end{aligned}
$$

Third, if the $j$-th $(j<n)$ minor failure occurred before time $T$, the accumulated repair cost till the $j$-th minor failure exceeds $L$ and the first serious failure occurs after time $S_{2 j}$, 
then the system will be replaced at time $S_{2 j}, j=1,2, \ldots$, $n-1$. Therefore, the probability of the third case is given by

$$
\begin{aligned}
& \sum_{j=1}^{n-1} P\left(N_{2}(T) \geq j, Y_{j-1}<L<Y_{j}, Z>S_{2 j}\right) \\
& \quad=\sum_{j=1}^{n-1}\left(G^{(j-1)}(L)-G^{(j)}(L)\right) P\left(S_{2 j} \leq T, Z>S_{2 j}\right) \\
& \quad=\sum_{j=0}^{n-2}\left(G^{(j)}(L)-G^{(j+1)}(L)\right) \int_{0}^{T} \overline{F_{z}}(t) P_{2 j}(t) q(t) \lambda(t) \mathrm{d} t
\end{aligned}
$$

Finally, if a serious failure occurs before time $T$ (i.e., $Z<T)$ and the accumulated repair cost before serious failure is less than $L$, then the system will be replaced at time $Z$. Therefore, the probability of the final case is given by

$$
\begin{aligned}
& \sum_{j=0}^{n-1} P\left(Z<T, N_{2}(z)=j, Y_{j}<L\right) \\
& \quad=\sum_{j=0}^{n-1} \int_{0}^{T} P_{2 j}(t) P\left(Y_{j}<L\right) \mathrm{d} F_{z}(t) \\
& \quad=\sum_{j=0}^{n-1} G^{(j)}(L) \int_{0}^{T} P_{2 j}(t) r_{z}(t) \overline{F_{z}}(t) \mathrm{d} t
\end{aligned}
$$

More specifically, we need the following assumptions:

1. The system is monitored continuously and failures are detected immediately.

2. All repairs and replacements are completed instantaneously.

3. Replacements are perfect and do not affect the system characteristics.

4. $\lambda(t)$ is a continuous and increasing function of $t$ with $\lambda(t) \rightarrow \infty$ as $t \rightarrow \infty$.

5. $G^{(n)}(y)$ and $H^{(n)}(w)$ are PF2 (a Polya frequency function of order 2).

6. The policy is for the steady-state case.

From Lemma 3.7 in Barlow and Proschan [27], it is known that $G^{(n)}(L)$ (or $H^{(n)}(K)$ ) is decreasing in $n$ for all $L($ or $K)>0$. In addition, we can observe that $G^{(n)}(L)$ (or $\left.H^{(n)}(K)\right)$ is PF2 if and only if $G^{(n)}(L) / G^{(n-1)}(L)$ (or $H^{(n)}(K) / H^{(n-1)}(K)$ ) is decreasing in $n$ for all $L$ (or $K)>0$ (Gottlieb [28], p. 749).

The cost of a preventive replacement is $C_{0}$, and the cost of a failure replacement is $C_{1}$. The inequality $C_{1}>C_{0}$ signifies that failure replacement is not a planned action, so it needs additional maintenance and restoration costs.

\subsection{Long-term expected cost per unit time}

For our model, let $V_{i}$ denote the length of the $i$-th replacement cycle for $i=1,2,3 \ldots$ and let $R_{i}$ denote the operating costs over the renewal interval $V_{i}$. Thus $\left\{\left(V_{i}, R_{i}\right)\right\}$ constitutes a renewal reward process. If $C(t)$ denotes the expected operating costs over the time interval $[0, t]$, it is well known that

$$
\lim _{t \rightarrow \infty} \frac{C(t)}{t}=\frac{E\left(R_{1}\right)}{E\left(V_{1}\right)}
$$

(see e.g. [Ross [29], p. 52 ]). Let $C(n, T, L)$ denote the righthand side of (11).

The expected length of a replacement cycle $E\left(V_{1}\right)$ depends on the situations and can be computed as follows:

$$
\begin{aligned}
E\left(V_{1}\right)= & {\left[T \times \overline{F_{z}}(T) \sum_{j=0}^{n-1} P_{2 j}(T) G^{(j)}(L)\right] } \\
& +\left[G^{(n-1)}(L) \int_{0}^{T} t \times \overline{F_{z}}(t) P_{2 n-1}(t) q(t) \lambda(t) \mathrm{d} t\right] \\
& +\left[\sum_{j=0}^{n-2}\left(G^{(j)}(L)-G^{(j+1)}(L)\right) \int_{0}^{T} t \times \overline{F_{z}}(t) P_{2 j}(t) q(t) \lambda(t) \mathrm{d} t\right] \\
& +\left[\sum_{j=0}^{n-1} G^{(j)}(L) \int_{0}^{T} t \times P_{2 j}(t) r_{z}(t) \overline{F_{z}}(t) \mathrm{d} t\right] \\
= & \sum_{j=0}^{n-1} G^{(j)}(L) \int_{0}^{T} \overline{F_{z}}(t) P_{2 j}(t) \mathrm{d} t
\end{aligned}
$$

If the replacement of the system is executed at time $T$, then the total cost will be $C_{0}+\sum_{i=1}^{N_{2}(T)} X_{i}$. And, if the replacement is executed at $n$-th minor failure, then the total cost will be $C_{0}+\sum_{i=1}^{n-1} X_{i}$. And, if the replacement is executed at $j$-th minor failure in which the accumulated repaired cost till the $j$-th minor failure exceeds $L$, then the total cost will be $C_{0}+\sum_{i=1}^{j-1} X_{i}, j=1,2, \ldots, n-1$. Furthermore, if the replacement is executed at the first serious failure, then the total cost will be $C_{1}+\sum_{i=1}^{N_{2}\left(W_{1}\right)} X_{i}$.

Therefore, the expected total cost during a replacement cycle $E\left(R_{1}\right)$ can be dervied as follows.

$$
\begin{aligned}
E\left(R_{1}\right)= & {\left[\overline{F_{z}}(T) \sum_{j=0}^{n-1} E\left[C_{0}+\sum_{i=1}^{j} X_{i}\right] P_{2 j}(T) G^{(j)}(L)\right] } \\
& +\left[G^{(n-1)}(L) \int_{0}^{T} E\left[C_{0}+\sum_{i=1}^{n-1} X_{i}\right] \times \overline{F_{z}}(t) P_{2 n-1}(t) q(t) \lambda(t) \mathrm{d} t\right] \\
& +\left[\sum_{j=1}^{n-1}\left(G^{(j-1)}(L)-G^{(j)}(L)\right)\right. \\
& \left.\times \int_{0}^{T} E\left[C_{0}+\sum_{i=1}^{j-1} X_{i}\right] \times \overline{F_{z}}(t) P_{2 j-1}(t) q(t) \lambda(t) \mathrm{d} t\right] \\
& +\left[\sum_{j=0}^{n-1} G^{(j)}(L) \int_{0}^{T} E\left[C_{1}+\sum_{i=1}^{j} X_{i}\right] P_{2 j}(t) r_{z}(t) \overline{F_{z}}(t) \mathrm{d} t\right] \\
= & C_{0}+\left(C_{1}-C_{0}\right)\left[\sum_{j=0}^{n-1} G^{(j)}(L) \int_{0}^{T} P_{2 j}(t) r_{z}(t) \overline{F_{z}}(t) \mathrm{d} t\right] \\
& +\mu_{x}\left[\sum_{j=1}^{n-1} G^{(j)}(L) \int_{0}^{T} \overline{F_{z}}(t) P_{2 j-1}(t) q(t) \lambda(t) \mathrm{d} t\right]
\end{aligned}
$$

Combining Eqs. (12) and (13), the long-term expected cost per unit time $C(n, T, L)$ can be obtained as follows: 


$$
C(n, T, L)=\frac{C_{0}+\left(C_{1}-C_{0}\right)\left[\sum_{j=0}^{n-1} G^{(j)}(L) \int_{0}^{T} P_{2 j}(t) r_{z}(t) \overline{F_{z}}(t) \mathrm{d} t\right]+\mu_{x}\left[\sum_{j=1}^{n-1} G^{(j)}(L) \int_{0}^{T} \overline{F_{z}}(t) P_{2 j-1}(t) q(t) \lambda(t) \mathrm{d} t\right]}{\sum_{j=0}^{n-1} G^{(j)}(L) \int_{0}^{T} \overline{F_{z}}(t) P_{2 j}(t) \mathrm{d} t} .
$$

\subsection{Optimization}

In the steady-state case, this problem is to find the values of $T$ and $n$ that minimize the function $C(n, T, L)$ given by Eq. (14). First, we shall minimize $C(n, T, L)$ with respect to $T$ for given $n$ and $L$. A necessary condition that a finite optimal $T$ minimizes $C(n, T, L)$ can be obtained by differentiating $C(n, T, L)$ with respect to $T$ and setting it equal to zero. Hence, we have that $\mathrm{d} C(n, T, L) / \mathrm{d} T=0$ holds if and only if

$$
U(n, T)=C_{0}
$$

where

$$
\begin{aligned}
U(n, T)= & A(n, T) \times \sum_{j=0}^{n-1} G^{(j)}(L) \int_{0}^{T} \overline{F_{z}}(t) P_{2, j}(t) \mathrm{d} t \\
& -\left(C_{1}-C_{0}\right)\left[\sum_{j=0}^{n-1} G^{(j)}(L) \int_{0}^{T} P_{2 j}(t) r_{z}(t) \overline{F_{z}}(t) \mathrm{d} t\right] \\
& -\mu_{x}\left[\sum_{j=1}^{n-1} G^{(j)}(L) \int_{0}^{T} \overline{F_{z}}(t) P_{2 j-1}(t) q(t) \lambda(t) \mathrm{d} t\right]
\end{aligned}
$$

and

$$
\begin{aligned}
A(n, T)= & \left(C_{1}-C_{0}\right) \times \frac{f_{z}(T)}{\overline{F_{z}}(T)}+\mu_{x} q(T) \lambda(T) \\
& \times \frac{\sum_{j=1}^{n-1} G^{(j)}(L) P_{2 j-1}(T)}{\sum_{j=0}^{n-1} G^{(j)}(L) P_{2 j}(T)}
\end{aligned}
$$

Next, we shall minimize $C(n, T, L)$ with respect to $n$ for given $T$ and $L$. When the inequalities $C(n+1, T, L) \geq C(n, T$, $L)$ and $C(n, T, L)<C(n-1, T, L)$ are both satisfied for some finite $n$, there exists an optimal $n^{*}$. In the derivation of these inequalities, we can see that the inequalities $C(n+1, T, L) \geq$ $C(n, T, L)$ and $C(n, T, L)<C(n-1, T, L)$ hold if and only if

$$
K(n, T) \geq C_{0} \quad \text { and } \quad K(n-1, T)<C_{0}
$$

where

$$
\begin{array}{ll}
K(n, T) & \\
\left(\left(C_{1}-C_{0}\right) B_{n}+\mu_{x} D_{n}\right) \times \sum_{j=0}^{n-1} G^{(j)}(L) \int_{0}^{T} \overline{F_{z}}(t) P_{2, j}(t) \mathrm{d} t & n=1,2,3, \ldots \\
-\left(C_{1}-C_{0}\right) \times \sum_{j=0}^{n-1} G^{(j)}(L) \int_{0}^{T} P_{2, j}(t) r_{z}(t) \overline{F_{z}}(t) \mathrm{d} t-\mu_{x} \times \sum_{j=1}^{n-1} G^{(j)}(L) \int_{0}^{T} \overline{F_{z}}(t) P_{2, j-1}(t) q(t) \lambda(t) \mathrm{d} t & \\
0 & n=0
\end{array}
$$

and

$$
\begin{aligned}
B_{n} & \left.=\int_{0}^{T} P_{2, n}(t) r_{z}(t) \overline{F_{z}}(t) \mathrm{d} t\right) / \int_{0}^{T} \overline{F_{z}}(t) P_{2, n}(t) \mathrm{d} t \\
D_{n} & =\int_{0}^{T} \overline{F_{z}}(t) P_{2, n-1}(t) q(t) \lambda(t) \mathrm{d} t / \int_{0}^{T} \overline{F_{z}}(t) P_{2, n}(t) \mathrm{d} t
\end{aligned}
$$

If $K(n, T)$ can be showed to be an increasing function of $n$ and $\lim n \rightarrow \infty K(n, T)>C_{0}$, then the optimal $n^{*}$ is finite and unique for all $T>0$. In order to show that $K(n, T)$ is an increasing function of $n$, the following Lemma 1 is needed.

Lemma 1 Under assumptions (a1) and (a2), the following results are true:

1. $\left.B_{n}=\int_{0}^{T} P_{2, n}(t) r_{z}(t) \overline{F_{z}}(t) \mathrm{d} t\right) / \int_{0}^{T} \overline{F_{z}}(t) P_{2, n}(t) \mathrm{d} t \quad$ is increasing in $n$ and $\lim _{n \rightarrow \infty} B_{n}=p(T) \lambda(T)$ for all $T>0$.

2. $D_{n}=\int_{0}^{T} \overline{F_{z}}(t) P_{2, n-1}(t) q(t) \lambda(t) \mathrm{d} t / \int_{0}^{T} \overline{F_{z}}(t) P_{2, n}(t) \mathrm{d} t \quad$ is increasing in $n$ and $\lim _{n \rightarrow \infty} D_{n}=q(T) \lambda(T)$ for any $T>0$.

The proof of Lemma 1 can be found in Chang et al [21]. We seek the optimal pair $n^{*}$ and $T^{*}$ that minimize $C(n, T$, $L$ ) in (10) under certain sufficient conditions.

Theorem 1 Under assumptions (4) and (6), if $\lambda(t)$ is an increasing and continuous function of $t$, there exists finite $n^{*}$ and $T^{*}$ that satisfy (15) and (18), respectively.

Proof It is evident that $U(n, 0) \equiv \lim _{T \rightarrow 0-}$ $U(n, T)=0<C_{0}$ and

$$
\begin{aligned}
U(n, \infty)= & \lim _{T \rightarrow \infty} U(n, T) \\
= & \lim _{T \rightarrow \infty} A(n, T) \times \sum_{j=0}^{n-1} G^{(j)}(L) \int_{0}^{\infty} \overline{F_{z}}(t) P_{2, j}(t) \mathrm{d} t \\
& -\left(C_{1}-C_{0}\right)\left[\sum_{j=0}^{n-1} G^{(j)}(L) \int_{0}^{\infty} P_{2 j}(t) r_{z}(t) \overline{F_{z}}(t) \mathrm{d} t\right] \\
- & \mu_{x}\left[\sum_{j=1}^{n-1} G^{(j)}(L) \int_{0}^{\infty} \overline{F_{z}}(t) P_{2 j-1}(t) q(t) \lambda(t) \mathrm{d} t\right] \\
= & \infty
\end{aligned}
$$


Since $\lim _{T \rightarrow \infty} \lambda(T)=\infty$ and $\lim _{T \rightarrow \infty} A(n, T)=\infty$. Hence, we can know that there exists at least one finite optimal $T^{*}$ such that $U(T, n)=C_{0}$, i.e., $T^{*}$ satisfies (11). Furthermore, if $\lambda(t)$ is strictly increasing and $\frac{\mathrm{d}}{\mathrm{d} t} U(n, T)=$ $A^{\prime}(n, T) \times \sum_{j=0}^{n-1} G^{(j)}(L) \int_{0}^{T} \overline{F_{z}}(t) P_{2 j}(t) \mathrm{d} t>0$, then $U(T, n)$ is a strictly increasing function of $t$, and hence, the optimal $T^{*}$ is unique.

In Eq. (19), we know that $K(0, T)=0<C_{0}$. If $\lim K(n, T)>C_{0}$, we can observe that there is a finite $n$ such that Eq. (18) is satisfied. Using Eq. (18) and Lemma 1, we have

$$
\begin{aligned}
K( & +1, T)-K(n, T)=\left(\left(C_{1}-C_{0}\right) B_{n+1}+\mu_{x} D_{n+1}\right) \\
& \times \sum_{j=0}^{n} G^{(j)}(L) \int_{0}^{T} \overline{F_{z}}(t) P_{2, j}(t) \mathrm{d} t \\
& -\left(C_{1}-C_{0}\right) \times \sum_{j=0}^{n} G^{(j)}(L) \int_{0}^{T} P_{2, j}(t) d F_{z}(t)-\mu_{x} \\
& \times \sum_{j=1}^{n} G^{(j)}(L) \int_{0}^{T} \overline{F_{z}}(t) P_{2, j-1}(t) q(t) \lambda(t) \mathrm{d} t \\
& -\left(\left(C_{1}-C_{0}\right) B_{n}+\mu_{x} D_{n}\right) \times \sum_{j=0}^{n-1} G^{(j)}(L) \int_{0}^{T} \overline{F_{z}}(t) P_{2, j}(t) \mathrm{d} t \\
& +\left(C_{1}-C_{0}\right) \times \sum_{j=0}^{n-1} G^{(j)}(L) \int_{0}^{T} P_{2, j}(t) d F_{z}(t)+\mu_{x} \\
& \times \sum_{j=1}^{n-1} G^{(j)}(L) \int_{0}^{T} \overline{F_{z}}(t) P_{2, j-1}(t) q(t) \lambda(t) \mathrm{d} t \\
= & {\left[\left(C_{1}-C_{0}\right)\left(B_{n+1}-B_{n}\right)+\mu_{x}\left(D_{n+1}-D_{n}\right)\right] } \\
& \times \sum_{j=0}^{n-1} G^{(j)}(L) \int_{0}^{T} \overline{F_{z}}(t) P_{2, j}(t) \mathrm{d} t
\end{aligned}
$$

By Lemma 1, we can know that $B_{n+1}-B_{n}>0$ and $D_{n+1}-D_{n}>0$ for any $T>0$; furthermore, $\sum_{j=0}^{n-1} G^{(j)}(L)$ $\int_{0}^{T} \overline{F_{z}}(t) P_{2, j}(t) \mathrm{d} t>0$, then $K(n+1, T)-K(n, T)>0$ for all $n$. We have that $K(n, T)$ is increasing in $n$. Thus, there exists a finite and unique $n^{*}$ that satisfies (18) for all $T>0$.

According to the above proof, we can know that there exists finite $T^{*}$ and $n^{*}$ that satisfy Eqs. (15) and (18), respectively.

\section{Special cases}

In this replacement model, it will be influenced by several parameters, such as $n, T, L$ and $p$. We have the following special cases:

Case 1 If $K=0, p(t)=p$ and $n=\infty$, then $\overline{F_{z}}(t)=$ $\exp \left(-\int_{0}^{t} p(x) \lambda(x) \mathrm{d} x\right)$ and this case is considered by Lai [18].
Case 2 If $L=\infty, q(t)=0$ and $n=\infty$, then $C(n, T, L)$ will be reduced to

$$
C(\infty, T, \infty)=\frac{C_{0}+\left(C_{1}-C_{0}\right)\left[1-\sum_{i=0}^{\infty} H^{(i)}(K) P_{1 i}(T)\right]}{\sum_{i=0}^{\infty} H^{(i)}(K) \int_{0}^{T} P_{1 i}(t) \mathrm{d} t}
$$

which is same as Eq. (3.11) in Nakagawa [1].

Case 3 If $T=\infty, L=\infty$, and $\mu_{x}=E(C)$, this is the case considered by Park [30].

\section{Numerical example}

In this example, we consider that the intensity function of shocks $\lambda(t)$ is taking as

$$
\lambda(t)=\lambda t^{\beta-1}, \quad \lambda>0, \quad \beta>1 .
$$

We assume that the shape parameter is set at $\beta=2$, so that $\lambda(t)=\lambda t$ is an increasing function of $t$. We also assume that $p(t)=p$ and $q(t)=q$ for all $t>0$.

Concerning the simultaneous optimization of both $T$ and $n$, that is, to determine the optimal policy, we use the following algorithm proposed in Badia and Berrade [31].

Step 1. Set $n=1$

Step 2. Find $T^{*}(n)$ and $T^{*}(n+1)$ by Eq. (15)

Step 3. If $C\left(n+1, T^{*}, L\right)<C\left(n, T^{*}, L\right)$, then proceed to Step 4; otherwise, advance to Step 5

Step 4. Set $n=n+1$, then go back Step 2

Step 5. Set $n^{*}=n$

The optimal policy turns out to be $\left(n^{*}, T^{*}\right)$. It is noted that the foregoing algorithm does not ensure a global minimum. Nevertheless, the algorithm can considerably reduce the search of the optimal values.

Two replacement costs $C_{0}$ and $C_{1}$ are 1,000 and 1,500, respectively. The amount of damage from consecutive type-I shocks is i.i.d. exponential random variables with finite mean $\mu_{d}=100$. And, the failure level of the system is set at $K=800$. The costs for consecutive minimal repairs are also i.i.d. exponential random variables with finite mean $\mu_{x}=50$. In addition, the cumulative repair cost limit $L$ is fixed to be 500 .

As $\lambda(t)=\lambda t$ is an increasing and continuous function of $t$, therefore, $K(n, T)$ is increasing in $n$ and $U(n, \infty)=\lim _{T \rightarrow \infty} U(n, T)=\infty$. Thus, the optimal $n^{*}$ and $T^{*}$ are finite and unique according to the conditions of Theorem 1 . The optimal period $T^{*}$, the optimal number $n^{*}$ and the minimum $C\left(n^{*}, T^{*}, L\right)$ with regard to different sets of the parameters values of $\lambda$ and $p$ will be showed in tables 1,2 , and 3 . From tables $1-3$, we have the following conclusions:

1. If the rate $\lambda$ of the function $\lambda(t)$ increases, then $\lambda(t)$ is more strongly increasing function of $t$. When the rate $\lambda$ 
Table 1. Optimal $T^{*}$ and $C\left(\infty, T^{*}, L\right)$ at different $\lambda$ and $p$ when $L / \mu_{x}=10, K / \mu_{d}=8$, and $n=\infty$.

\begin{tabular}{|c|c|c|c|c|c|c|c|c|c|c|}
\hline \multirow[b]{3}{*}{$\lambda$} & \multicolumn{10}{|c|}{$p(q)$} \\
\hline & \multicolumn{2}{|c|}{$0.3(0.7)$} & \multicolumn{2}{|c|}{$0.4(0.6)$} & \multicolumn{2}{|c|}{$0.5(0.5)$} & \multicolumn{2}{|c|}{$0.6(0.4)$} & \multicolumn{2}{|c|}{$0.7(0.3)$} \\
\hline & $T^{*}$ & $C\left(\infty, T^{*}, L\right)$ & $T^{*}$ & $C\left(\infty, T^{*}, L\right)$ & $T^{*}$ & $C\left(\infty, T^{*}, L\right)$ & $T^{*}$ & $C\left(\infty, T^{*}, L\right)$ & $T^{*}$ & $C\left(\infty, T^{*}, L\right)$ \\
\hline 1.0 & 6.4268 & 293.2890081 & 5.6248 & 294.5745545 & 5.1244 & 299.0381788 & 4.7681 & 304.9960242 & 4.4910 & 311.7159505 \\
\hline 1.5 & 5.2475 & 359.2042083 & 4.5925 & 360.7786751 & 4.1841 & 366.2454760 & 3.8931 & 373.5423163 & 3.6668 & 381.7725118 \\
\hline 2.0 & 4.5448 & 414.7732929 & 3.9773 & 416.5913304 & 3.6235 & 422.9038484 & 3.7716 & 431.3295140 & 3.1757 & 440.8329249 \\
\hline 2.5 & 4.0648 & 463.7306391 & 3.5574 & 465.7632665 & 3.2410 & 472.8208765 & 3.0156 & 482.2410573 & 2.8404 & 492.8661930 \\
\hline
\end{tabular}

Table 2. Optimal $n^{*}$ and $C\left(n^{*}, \infty, L\right)$ at different $\lambda$ and $p$, when $L / \mu_{x}=10, K / \mu_{d}=8$, and $T=\infty$.

\begin{tabular}{|c|c|c|c|c|c|c|c|c|c|c|}
\hline \multirow[b]{3}{*}{$\lambda$} & \multicolumn{10}{|c|}{$p(q)$} \\
\hline & \multicolumn{2}{|r|}{$0.3(0.7)$} & \multicolumn{2}{|r|}{$0.4(0.6)$} & \multicolumn{2}{|r|}{$0.5(0.5)$} & \multicolumn{2}{|r|}{$0.6(0.4)$} & \multicolumn{2}{|r|}{$0.7(0.3)$} \\
\hline & $n^{*}$ & $C\left(n^{*}, \infty, L\right)$ & $n^{*}$ & $C\left(n^{*}, \infty, L\right)$ & $n^{*}$ & $C\left(n^{*}, \infty, L\right)$ & $n^{*}$ & $C\left(n^{*}, \infty, L\right)$ & $n^{*}$ & $C\left(n^{*}, \infty, L\right)$ \\
\hline 1.0 & 12 & 292.9909184 & 9 & 294.9119827 & 7 & 301.3978537 & 5 & 310.5921124 & 4 & 321.3561456 \\
\hline 1.5 & 12 & 358.8391246 & 9 & 361.1919384 & 7 & 369.1354753 & 5 & 380.3960969 & 4 & 393.5792913 \\
\hline 2.0 & 12 & 414.3517304 & 9 & 417.0685255 & 7 & 426.2409323 & 5 & 439.2435780 & 4 & 454.4662197 \\
\hline 2.5 & 12 & 463.2593182 & 9 & 466.2967872 & 7 & 476.5518497 & 5 & 491.0892494 & 4 & 508.1086802 \\
\hline
\end{tabular}

Table 3. Optimal $n^{*}, T^{*}$ and $C\left(n^{*}, T^{*}, L\right)$ at different $\lambda$ and $p$, when $L / \mu_{x}=10$ and $K / \mu_{d}=8$.

\begin{tabular}{|c|c|c|c|c|c|c|c|c|c|c|c|c|c|c|c|}
\hline \multirow[b]{3}{*}{$\lambda$} & \multicolumn{15}{|c|}{$p(q)$} \\
\hline & \multicolumn{3}{|c|}{$0.3(0.7)$} & \multicolumn{3}{|c|}{$0.4(0.6)$} & \multicolumn{3}{|c|}{$0.5(0.5)$} & \multicolumn{3}{|c|}{$0.6(0.4)$} & \multicolumn{3}{|c|}{$0.7(0.3)$} \\
\hline & $T^{*}$ & $n^{*}$ & $\begin{array}{c}C\left(n^{*}, T^{*},\right. \\
L)\end{array}$ & $T^{*}$ & $n^{*}$ & $\begin{array}{c}C\left(n^{*}, T^{*},\right. \\
L)\end{array}$ & $T^{*}$ & $n^{*}$ & $\begin{array}{c}C\left(n^{*}, T^{*}\right. \\
L)\end{array}$ & $T^{*}$ & $n^{*}$ & $\begin{array}{c}C\left(n^{*}, T^{*}\right. \\
L)\end{array}$ & $T^{*}$ & $n^{*}$ & $\begin{array}{c}C\left(n^{*}, T^{*}\right. \\
L)\end{array}$ \\
\hline 1.0 & 6.6706 & 13 & 292.9283 & 5.8182 & 10 & 293.9779 & 5.2060 & 9 & 298.5935 & 4.8365 & 7 & 304.6853 & 4.5232 & 6 & 311.5753 \\
\hline 1.5 & 5.4461 & 13 & 358.7625 & 4.7506 & 10 & 360.0479 & 4.2507 & 9 & 365.7009 & 3.9490 & 7 & 373.1618 & 3.6932 & 6 & 381.6002 \\
\hline 2.0 & 4.7166 & 13 & 414.2632 & 4.1141 & 10 & 415.7476 & 3.6811 & 9 & 422.2751 & 3.4198 & 7 & 430.8901 & 3.1983 & 6 & 440.6340 \\
\hline 2.5 & 4.2185 & 13 & 463.1603 & 3.6798 & 10 & 464.8199 & 3.2926 & 9 & 472.1178 & 3.0588 & 7 & 481.7498 & 2.8607 & 6 & 492.6438 \\
\hline
\end{tabular}

increases, we can know that the optimal $T^{*}$ decreases but the minimum $C\left(\infty, T^{*}, L\right)$ increases in table 1 . These results in table 1 are the same as Chien et al [19] and Chien et al [20]. As the rate $\lambda$ increases, it implies that the arriving shocks occur more frequently, the replacement period must be shorter to prevent the occurrence of random failures.

2 . When the rate $\lambda$ increases, we can know that the optimal $n^{*}$ is all the same but the minimum $C\left(n^{*}, \infty, L\right)$ increases in table 2. Because the accumulation of damages is faster than the accumulation of repair costs, then the occurrence of serious failure is earlier than a preventive replacement. We must want to avoid this situation, the optimal $n^{*}$ remains the same in tables 2 and 3 , even when $\lambda$ is decreasing. Chang et al [21] has the same result that the optimal $n^{*}$ does not depend at all on the rate $\alpha$ of increase in $r(t)$.

3. When $p$ increases (i.e., $q$ decreases), we can see that the optimal $T^{*}$ decreases but the minimum $C\left(\infty, T^{*}, L\right)$ increases in table 1 and the optimal $n^{*}$ decreases but the minimum $C\left(n^{*}, \infty, L\right)$ increases in table 2 . This is intuitive as the lower probability of replacement leads to the lower expected operating cost. The greater $p$ implies that serious failure more easy to occur, so the replacement period must be shorter also to prevent the occurrence of serious failures. This result is the same as Sheu et al [25].

4. In table 3 , we can see the same results when $\lambda$ or $p$ increases, the minimum $C\left(n^{*}, T^{*}, L\right)$ is less than $C(\infty$, $\left.T^{*}, L\right)$ in table 1 and $C\left(n^{*}, \infty, L\right)$ in table 2 at the same sets of the parameters values. So, the optimal pair $\left(n^{*}\right.$, $\left.T^{*}\right)$ policy gets better results.

Furthermore, the optimal period $T^{*}$, the optimal number $n^{*}$ and the minimum $C\left(n^{*}, T^{*}, L\right)$ with regard to different sets of the ratios $L / \mu_{x}$ and $K / \mu_{d}$ will be showed in tables 4 . From table 4, we have the following conclusions:

5. When the ratio $L / \mu_{x}$ increases, i.e. it allows more minor failures before replacement, we can see that the optimal 
Table 4. Optimal $n^{*}, T^{*}$ and $C\left(n^{*}, T^{*}, L\right)$ at different values of $L / \mu_{x}$ and $K / \mu_{d}$ when $p=0.5, \lambda=2$.

\begin{tabular}{|c|c|c|c|c|c|c|c|c|c|c|c|c|}
\hline \multirow[b]{3}{*}{$K / \mu_{d}$} & \multicolumn{12}{|c|}{$L / \mu_{x}$} \\
\hline & \multicolumn{3}{|c|}{6} & \multicolumn{3}{|c|}{8} & \multicolumn{3}{|c|}{10} & \multicolumn{3}{|c|}{12} \\
\hline & $T^{*}$ & $n^{*}$ & $C\left(n^{*}, T^{*}, L\right)$ & $T^{*}$ & $n^{*}$ & $C\left(n^{*}, T^{*}, L\right)$ & $T^{*}$ & $n^{*}$ & $C\left(n^{*}, T^{*}, L\right)$ & $T^{*}$ & $n^{*}$ & $C\left(n^{*}, T^{*}, L\right)$ \\
\hline 6 & 3.4111 & 8 & 472.9354655 & 3.3722 & 8 & 468.5900622 & 3.3572 & 8 & 467.3627714 & 3.3516 & 8 & 467.0349010 \\
\hline 8 & 3.7626 & 9 & 430.3957109 & 3.7059 & 9 & 424.2111362 & 3.6811 & 9 & 422.2750384 & 3.6711 & 9 & 421.7024767 \\
\hline 10 & 4.1283 & 9 & 404.3021183 & 4.0623 & 9 & 395.9951381 & 4.0337 & 9 & 393.1218528 & 4.0220 & 9 & 392.1814548 \\
\hline 12 & 4.4612 & 10 & 387.7770145 & 4.3751 & 10 & 377.2944941 & 4.3336 & 10 & 373.3471058 & 4.3147 & 10 & 371.9349854 \\
\hline
\end{tabular}

$T^{*}$ and the minimum $C\left(n^{*}, T^{*}, L\right)$ decreases. Under the fixed ratio $K / \mu_{d}$, the decreasing magnitudes of the optimal $T^{*}$ and $C\left(n^{*}, T^{*}, L\right)$ are significantly smaller than the increments of $L / \mu_{x}$.

6. When the ratio $K / \mu_{d}$ increases, i.e. it allows more type-I shocks to occur before serious failure, we can see that the optimal $T^{*}$ increases but the minimum $C\left(n^{*}, T^{*}, L\right)$ decreases. The variations in the optimal $T^{*}$ and $C\left(n^{*}, T^{*}\right.$, $L$ ) with regard to $K / \mu_{d}$ are significantly larger than that of $L / u_{x}$. So, the ratio $K / \mu_{d}$ is more important than the ratio $L / \mu_{x}$ when we are going to determine the optimal replacement policy.

\section{Conclusions}

In this paper, a bivariate replacement policy $(n, T)$ with cumulative repair-cost limit under a standard cumulative damage model is introduced. The long-term expected cost per unit time $C(n, T, L)$ by incorporating costs due to replacement and minimal repair is derived. This research verifies that under certain conditions, the optimal $n^{*}$ and the optimal $T^{*}$ to minimize $C(n, T, L)$ is finite and unique under fixed the values of $L$ and $K$, respectively. This work can extend to consider multi-unit system or include the concept of imperfect repair.

\section{Acknowledgments}

This research was supported by the Ministry of Science and Technology of Taiwan, under Grant No. MOST 104-2410H-218 -012 -MY2.

\section{References}

[1] Nakagawa T 2007 Shock and damage models in reliability theory. London: Springer-Verlag

[2] Feldman R M 1976 Optimal replacement with semi-Markov shock models. J. Appl. Probability 13: 108-117

[3] Nakagawa T 1976 On a replacement problem of a cumulative damage model. Operat. Res. 27(4): 895-900

[4] Satow T, Teramoto K and Nakagawa T 2000 Optimal replacement policy for a cumulative damage model with time deterioration. Math. Comput. Model., 31(10-12): 313-319

[5] Taylor H M 1975 Optimal replacement under additive damage and other failure models. Naval Res. Logist. 22(1): 1-18

[6] Mizuno N 1986 Generalized mathematical programming for optimal replacement in a Semi-Markov shock model. Operat. Res. 34: 790-795

[7] Nakagawa T 1980 A summary of imperfect preventive maintenance policies with minimal repair. RAIRO Operat. Res. 14: 249-255

[8] Qian C, Nakamura S and Nakagawa T 1999 Cumulative damage model with two kinds of shocks and its application to the backup policy. J. Operat. Res. Soc. Jpn 42: 501-511

[9] Perry D 2000 Control limit policies in a replacement model with additive phase-type distributed damage and linear restoration. Operat. Res. Lett. 27: 127-134

[10] Nakagawa T 1984 A summary of discrete replacement policies. Eur. J. Operat. Res. 17: 382-392

[11] Nakagawa T and Kijima M 1989 Replacement policies for a cumulative damage model. IEEE Trans. Reliab. 38(3): 581-584

[12] Satow T and Nakagawa T 1997 Three replacement models with two kinds of damage. Microelectron. Reliab. 37(6): 909-913

[13] Kijima M and Nakagawa T 1991 A cumulative damage shock model with imperfect preventive maintenance. Naval Res. Logist. 38(2): 145-156

[14] Qian C, Nakamura S and Nakagawa T 2003 Replacement and minimal repair policies for a cumulative damage model with maintenance. Comput. Math. Appl. 46(7): 1111-1118

[15] Qian C H, Ito K and Nakagawa T 2005 Optimal preventive maintenance policies for a shock model with given damage level. J. Quality Maintenance Eng. 11(3): 216-227

[16] Ito K and Nakagawa T 2011 Comparison of three cumulative damage models. Quality Technol. Quantitative Manag. 8(1): $57-66$

[17] Zhao X, Zhang H, Qian C, Nakagawa T and Nakamura S 2012 Replacement models for combining additive independent damages. Int. J. Performability Eng. 8: 91-100

[18] Lai M T 2007 A periodical replacement model based on cumulative repair cost limit. Appl. Stochastic Models Business Ind. 26: 455-464

[19] Chien Y H, Sheu S H, and Chang C C 2009 Optimal agereplacement time with minimal repair based on cumulative repair cost limit and random lead time. Int. J. Syst. Sci. 40: 703-715

[20] Chien Y H, Chang C C and Sheu S H 2010 optimal agereplacement model with age-dependent type of failure and 
random lead time based on a cumulative repair-cost limit policy. Ann. Operat. Res. 181: 723-744

[21] Chang C C, Sheu S H and Chen Y L 2010 Optimal number of minimal repairs before replacement based on a cumulative repair-cost limit policy. Comput. Ind. Eng. 59: 603-610

[22] Chang C C, Sheu S H and Chen Y L 2013 Optimal replacement model with age-dependent failure type based on a cumulative repair-cost limit policy. Appl. Math. Model. 37: 308-317

[23] Sheu S, Chang C C, Chen Y L and Zhang Z G 2010 A periodic replacement model based on cumulative repair-cost limit for a system subjected to shocks. IEEE Trans. Reliab. 59: 374-382

[24] Sheu S H, Chang C C, Zhang Z G and Chien Y H 2012 A note on replacement policy for a system subject to non-homogeneous pure birth shocks. Eur. J. Operat. Res. 216: 503-508

[25] Sheu S H, Chen Y L, Chang C C and Zhang Z G 2013 Extended optimal replacement policy for a system subject to non-homogeneous pure birth shocks. Comput. Ind. Eng. 64: 573-579

[26] Savits T H 1988 Some multivariate distributions derived from a non-fatal shock model. J. Appl. Probability 25: 383-390

[27] Barlow R E and Proschan F 1975 Statistical theory of reliability and life testing probability models. Holt, Rinehart and Winston, New York

[28] Gottlieb G 1980 Failure distributions of shock models. $J$. Appl. Probability 25: 745-752

[29] Ross S M 1983 Applied probability models with optimization applications. Holden-Day, San Francisco

[30] Park K S 1987 Optimal number of minor failures before replacement. Int. J. Syst. Sci. 18: 333-337

[31] Badia F D and Berrade M D 2009 Opportunity-based age replacement for a system under two types of failures. In: Martorell et al (eds) Safety, reliability and risk analysis: Theory, methods and applications. Taylor \& Francis Group, London, 575-580 